\title{
APLICAÇÃO DE KCN E LINAMARINA E A INCOMPATIBILIDADE DE ENXERTIA POR TRANSLOCAÇÃO NO CLONE DE SERINGUEIRA IPA 1
}

\author{
Larissa Alexandra Cardoso Moraes ${ }^{1 *}$; Vicente Haroldo de Figueiredo Moraes ${ }^{1}$; Paulo \\ Roberto de Camargo e Castro \\ ${ }^{1}$ Embrapa Amazonia Ocidental, C.P. 319 - CEP: 69011-970 - Manaus, AM. \\ 'Depto. de Ciências Biológicas - USP/ESALQ, C.P. 9 - CEP: 13418-900 - Piracicaba, SP. \\ *Autor correspondente <larissa@cpaa.embrapa.br>
}

RESUMO: Para caracterizar evidências indiretas do envolvimento dos glicosídeos cianogênicos da seringueira, cujo principal componente é a linamarina, no desenvolvimento dos sintomas de incompatibilidade de enxertia por translocação apresentados pelo clone de painel IPA 1 (Hevea brasiliensis) em enxertos de copa com vários clones de outras espécies de Hevea, tentou-se sua reprodução através da aplicação de soluções com concentrações de cianeto de potássio $(\mathrm{KCN})$ e linamarina no caule do clone IPA 1 e dos clones Fx 4098 e Fx 3864, que não apresentam reação de incompatibilidade com os mesmos clones incompatíveis com o IPA 1. A aplicação de soluções de $\mathrm{KCN}$ no caule provocaram rápida coagulação do látex nos laticíferos do IPA 1 com concentrações muito baixas de KCN e mais altas no Fx 4098. No Fx 3864, não houve efeito, exceto leve redução de escoamento com a dose mais alta. Os tratamentos com KCN causaram necrose apenas próximo ao local das aplicações. Com soluções de linamarina, a necrose, também precedida pela coagulação do látex, ocorreu em maior extensão do caule, o que indica maior mobilidade de linamarina, que do KCN. O IPA 1 mostrou-se, novamente, mais sensível às concentrações mais baixas de linamarina, com doses mais altas provocando os mesmos efeitos no Fx 4098 e sem ocorrência de efeitos no Fx 3864. Nos tratamentos com linamarina observou-se o acúmulo de HCN livre na casca do caule do clone IPA 1, responsável pelos sintomas observados. A incompatibilidade com o IPA 1 é provocada pela translocação de glicosídios cianogênicos das copas enxertadas no IPA 1, que não é dotado de suficiente capacidade de remoção do HCN liberado na hidrólise desses glicosídios, tendo o Fx 3864 apresentado desintoxicação mais eficiente.

Palavras-chave: Hevea brasiliensis, glicosídio cianogênico, cianogênese

\section{KCN AND LINAMARIN APPLICATIONS AND THE TRANSLOCATION INCOMPATIBILITY OF CROWN CLONES OF Hevea sp. BUDDED ONTO IPA 1}

\begin{abstract}
To further evaluate previous indirect evidences of a role for the rubber tree cyanogenic glycosides, of which the main component is linamarin, this study evaluated the translocation incompatibility of the clone IPA 1 (Hevea brasiliensis) with budded crowns of clones of other species. Solutions of KCN were first applied, which caused rapid coagulation of the latex in the laticiphers of IPA 1, at very low concentrations, the same effect being observed in Fx 4098 at higher concentrations. The clone Fx 3864 presented no effect, only a slight reduction of the latex flow at the highest concentration. With linamarin solutions the necrosis, again preceded by latex coagulation, spread longer along the stem, indicating a higher mobility of linamarin than $\mathrm{KCN}$. The clone IPA 1 behaved again sensitive to the lower concentrations of linamarin, with the higher concentrations causing the same effects on Fx 4098 and no effect on Fx 3864 . With linamarin a build up of free HCN was detected in the bark of IPA 1 . The incompatibility with IPA 1 is caused by the translocation of cianogenic glycosides from the budded crowns to the stem which is deprived of sufficient capacity to remove the HCN liberated by the hydrolisis of cyanogenic glicosides, while the Fx 3864 presented a very efficient detoxifying capacity.
\end{abstract}

Key words: Hevea brasiliensis, cianogenic glucosides, cyanogenesis

\section{INTRODUÇÃO}

A utilização da enxertia de copa com clones resistentes ao Microcyclus ulei (P. Henn.) v. Arx. viabiliza o desenvolvimento da heveicultura na região Amazônica, cujas condições são propícias a surtos epidêmicos severos do mal-das-folhas, enfermidade causada por esse fungo (Moraes, 1997), sendo rara a ocorrência de incompatibilidade de enxertia no gênero Hevea, mesmo entre clones de espécies diferentes, não se tendo registros de incompatibilidade por translocação tal como definida por Mosse (1962) e Hartmann et al. (1990).

No entanto, em ensaio de teste de clones de copa, utilizando-se plantas jovens, tal tipo de incompatibilidade foi constatada, recentemente, em combinações cujo clone de painel foi o IPA 1 e os de 
copa, os provenientes de $H$. pauciflora e $H$. guianensis var. marginata (Moraes, 1999).

O primeiro sintoma da incompatibilidade é a ausência do escoamento do látex no caule do IPA 1, abaixo da união do enxerto, devida à coagulação do látex nos laticíferos. Segue-se a necrose da casca do IPA 1, em enxertos com três a quatro lançamentos foliares e a morte do enxerto após o anelamento completo da casca (Moraes, 1999).

Combinações compatíveis foram obtidas utilizando como painel o clone IPA 1 e como copa, clones de $H$. nitida (Moraes, 1999), cujo potencial cianogênico é baixo (Lieberei, 1988), o que conduziu à hipótese de que a incompatibilidade dos clones de copa com IPA 1 estaria relacionada com a cianogênese. Além de $H$. nitida, mostraram-se também compatíveis os clones PUA 5 e PUA 9 ( $H$. pauciflora) e F 4512 ( $H$. benthamiana), com baixo potencial cianogênico de acordo com Lieberei (1988).

A cianogênese, que é a produção de ácido cianídrico ( $\mathrm{HCN})$ por organismos vivos, é um fenômeno relativamente comum em plantas superiores, aproximando-se de 2000 o número de espécies com conhecida capacidade cianogênica (Conn, 1980). A liberação do $\mathrm{HCN}$ ocorre quando a planta sofre algum ferimento. Em plantas intactas, as enzimas que promovem a quebra dos glicosídios cianogênicos, chamadas genericamente de $\beta$-glicosidases, encontram-se separadas destes por compartimentação (Conn, 1980).

O envolvimento de translocação de compostos cianogênicos no desenvolvimento de incompatibilidade de enxertia já foi descrito por Gur (1957). Nesse caso, em enxertos de marmeleiro sob pereira, observou-se que o glicosídio cianogênico prunasina ocorre no marmeleiro e não na pereira. Nas combinações incompatíveis há, na casca do caule da pereira, hidrólise mais rápida do glicosídio translocado provocando o aparecimento de distúrbios anatômicos causados pelo acúmulo de $\mathrm{HCN}$, com bloqueio do sistema condutor do floema.

No gênero Hevea, os glicosídios cianogênicos mais comumente encontrados são linamarina (2hidroxisobutironitrila- $\beta$-D-glucopiranosídeo) e (R)lotaustralina [(R)-(2-hidroxi-2-metilbutironitrila- $\beta$-Dglucopiranosídeo)], observando-se valores para a quantidade de lotaustralina sempre inferiores aos de linamarina, não ultrapassando $3 \%$ do conteúdo total de glicosídios cianogênicos (Lieberei et al., 1986) O conteúdo desses compostos em Hevea varia muito entre espécies e em uma única planta varia com o órgão, bem como com o estádio de desenvolvimento deste (Lieberei, 1988).

A função biológica mais freqüentemente atribuída à cianogênese em plantas é a de proteção contra herbívoros, devido às propriedades tóxicas do $\mathrm{HCN}$ liberado pelo tecido vegetal que sofreu ferimento (Kakes, 1990). No entanto, o papel que os compostos cianogênicos desempenham em plantas intactas, é ainda bastante controverso, sendo considerados por Selmar et al. (1990) como capazes de agirem como armazenadores de nitrogênio em sua forma reduzida. Em Hevea o mecanismo que permite a incorporação do nitrogênio no metabolismo geral da planta, promovendo a desintoxicação, é a fixação enzimática catalisada pela $\beta$ cianoalaninasintase (Lieberei, 1988). Nesse processo o $\mathrm{HCN}$ e a cisteína reagem para formar sulfeto de hidrogênio e $\beta$-cianoalanina (Floss et al., 1965; Blumenthal, et al., 1968; Castric et al., 1972), esta podendo ser metabolizada para asparagina, (Miller \& Conn, 1980).

Em plântulas de seringueira a translocação de glicosídios cianogênicos do endosperma da semente para as folhas e raízes, onde podem ser metabolizados disponibilizando o nitrogênio, foi constatada por Selmar (1993). Essa translocação ocorre após glicolização da linamarase, produzindo o diglicosídio linustatina, que não sofre hidrólise por ação da linamarase, $\beta$-glicosidase presente no apoplasma e responsável pela hidrólise de todos os $\beta$-glicosídios e $\beta$-galactosídios que ocorrem no gênero Hevea.

Em plantas adultas de seringueira ocorre uma maior concentração de compostos cianogênicos nas folhas jovens, não tendo sido constatada ainda sua translocação para outras partes da planta, o que poderia ocorrer de forma semelhante ao observado em plântulas.

Para verificar a possibilidade de os sintomas observados nas combinações incompatíveis terem sido provocados pela translocação dos glicosídios cianogênicos do clone de copa para o clone de painel, tentou-se a reprodução desses sintomas através da aplicação de soluções com diferentes concentrações de cianeto de potássio (KCN) e linamarina no caule do IPA 1 e dos clones Fx 4098 e Fx 3864, que não apresentam reação de incompatibilidade com os mesmos clones incompatíveis com o IPA 1.

\section{MATERIAL E MÉTODOS}

\section{Localização}

As coletas e as análises dos materiais vegetais foram realizadas no Centro de Pesquisa Agroflorestal da Amazônia Ocidental (Embrapa-CPAA), localizado no município de Manaus, Estado do Amazonas.

\section{Aplicação de $\mathrm{KCN}$, em dose única}

As aplicações foram feitas em ramos de sete a nove meses, com cerca de $1 \mathrm{~cm}$ de diâmetro no entrenó do penúltimo lançamento, brotados do caule principal decapitado, de plantas de jardim clonal, do clone de painel IPA $1(H$. brasiliensis). Este apresenta sintomas de incompatibilidade quando enxertado com clones de copa provenientes de $H$. pauciflora e $H$. guianensis var. marginata, e dos clones de painel Fx 4098 e Fx 3864 $(H$. brasiliensis) que são compatíveis com essas mesmas copas.

O KCN foi aplicado em solução aquosa com dimetilsulfóxido (DMSO), 0,05\%, em duas repetições de 
cada uma das seguintes concentrações em $\mathrm{mg} \mathrm{L}^{-1}$ : 0,0064; 0,032; 0,16; 0,8; 4,0; 20,0 e 100,0.

Para a aplicação das soluções, os ramos foram cortados abaixo do penúltimo lançamento com folhas maduras, com retirada dos coágulos de látex e certificação da parada completa de escoamento.

Em cada ramo cortado foi feita uma só aplicação de $2 \mathrm{~mL}$ de cada solução, contidos em segmentos de 5 $\mathrm{cm}$ de tubo plástico de paredes semi-elásticas, com 1 $\mathrm{cm}$ de diâmetro interno, tendo cerca de $1,5 \mathrm{~cm}$ inseridos ao redor do caule, a partir do topo cortado, com vedação de fita veda rosca entre a parede do tubo e a epiderme do caule. Após a aplicação da solução e a verificação da ausência de vazamento, os tubos foram cobertos com papel alumínio.

No clone Fx 4098 foi aplicada, adicionalmente, a mesma série de diluições de $\mathrm{KCN}$ em solução aquosa de DMSO 0,05\%, acrescida de sacarose $0,1 \mathrm{~mol} \mathrm{~L}^{-1}$, para verificar a ocorrência de um possível efeito de cotransporte.

A verificação da exsudação do látex foi feita por puncturas com estilete de $1 \mathrm{~mm}$ de diâmetro. Ao final das observações foram feitos cortes da casca a mão livre, corados em Sudam III, sem prévia fixação, para observação microscópica.

\section{Aplicação contínua de KCN}

As aplicações foram feitas como na aplicação de uma única dose de $2 \mathrm{~mL}$ por planta, descrita no item anterior, mantendo-se a solução nos tubos com reaplicações. Foram testadas as seguintes concentrações de $\mathrm{KCN} 0,032 \mathrm{mg} \mathrm{L}^{-1}$ e $0,16 \mathrm{mg} \mathrm{L}^{-1}$, no clone IPA 1, e 0,16 $\mathrm{mg} \mathrm{L}^{-1}$ e $20 \mathrm{mg} \mathrm{L}^{-1}$, no Fx 4098.

\section{Aplicação de linamarina}

A técnica de aplicação da linamarina foi idêntica à da aplicação contínua de $\mathrm{KCN}$, porém com volume inicial de $3 \mathrm{~mL}$, durante cinco dias. Foram também registrados os efeitos sobre o escoamento do látex, a ocorrência de sintomas externos de necrose na casca e feitas observações microscópicas em cortes de casca a mão livre. Foi incluído um tratamento testemunha com solução aquosa de DMSO 0,05\%. As concentrações testadas de linamarina foram: 0,04; 0,2; 1,0; 5,0; 25,0 e $125,0 \mathrm{mg} \mathrm{L}^{-1}$.

\section{Ação do cianeto na coagulação do látex "in vitro"}

Foi coletado látex em frascos de vidro de $50 \mathrm{~mL}$ de plantas adultas dos clones Fx 3864, Fx 4098 e IPA 1 , sangradas duas vezes por semana em meia espiral, sem estimulação, que foram circundados com gelo em copo de plástico e transportados em caixa de poliestireno.

$\mathrm{Em} 20 \mathrm{~mL}$ do látex de cada clone, previamente equilibrado com a temperatura ambiente, foi aplicada, com bureta, uma solução de $\mathrm{KCN}, 300 \mathrm{mmol} \mathrm{L}^{-1}$, com agitação vigorosa a cada aplicação de $0,1 \mathrm{~mL}$, até que se verificasse a coagulação, dentro de 20 segundos após a aplicação de cada alíquota, registrando-se o volume consumido da solução de KCN.

\section{Concentração de HCN livre nos caules submetidos a diferentes tempos de exposição à solução de linamarina}

A técnica de aplicação da linamarina foi a mesma descrita para o item anterior, utilizando-se apenas a concentração de $6 \mathrm{mg} \mathrm{L}^{-1}$, em solução aquosa de DMSO, 0,05\%. As aplicações foram feitas em caules jovens dos clones IPA 1 e Fx 3864, com duas repetições para cada tratamento, sendo considerada cada repetição uma planta e os tratamentos os diferentes tempos de exposição do caule à ação da linamarina: 6, 12, 16 e 24 horas e ainda um tratamento testemunha, sem aplicação de linamarina. 0 delineamento utilizado foi o inteiramente casualizado em esquema fatorial 4 (horas) $\times 2$ (clones), com duas repetições. Os resultados foram submetidos à análise de variância, teste $\mathrm{F}$ e regressão polinomial a $1 \%$ de probabilidade.

\section{Preparo da amostra}

O método utilizado para extração foi adaptado de Cooke (1978) e Selmar (1986). Os caules foram coletados nos tempos determinados para cada tratamento. No laboratório, retirou-se $1 \mathrm{~g}$ de casca, a partir da ponta do caule que estava em contato com a solução de linamarina. As cascas foram picadas e moídas em gral com pistilo pré resfriados com a adição de $4 \mathrm{~mL}$ de solução também pré resfriada de ácido ortofosfórico, 0,067 $\mathrm{mol} \mathrm{L}^{-1}$, levando o $\mathrm{pH}$ dos extratos para 2,81, correspondendo à faixa de inativação da $\beta$-glicosidase (Cooke, 1978), e de $500 \mathrm{mg}$ de areia lavada com solução de ácido clorídrico, $10 \%$, solução sulfocrômica e, por fim, água destilada.

O macerado obtido foi centrifugado a $20.000 \mathrm{~g}$ por 14 minutos, entre zero e seis graus centígrados. O sobrenadante foi então transferido para frasco de vidro com capacidade para $30 \mathrm{~mL}$.

\section{Determinação de HCN livre}

Do sobrenadante obtido após centrifugação, foi retirado $0,1 \mathrm{~mL}$ para outro frasco idêntico ao anterior, ao qual adicionou-se $0,45 \mathrm{~mL}$ de $\mathrm{NaH}_{2} \mathrm{PO}_{4},\left(0,067 \mathrm{~mol} \mathrm{~L}^{-1}\right)$, $0,6 \mathrm{~mL}$ de $\mathrm{NaOH}\left(0,2 \mathrm{~mol} \mathrm{~L}^{-1}\right)$ e $3,85 \mathrm{~mL}$ de água destilada, num total de $5 \mathrm{~mL}$. Para o branco foi utilizado $0,1 \mathrm{~mL}$ do sobrenadante adicionado de $0,45 \mathrm{~mL}$ de $\mathrm{NaH}_{2} \mathrm{PO}_{4}$, completando-se o volume para $5 \mathrm{~mL}$ com água destilada.

A seguir, foram acrescentados os reagentes do kit Merck Spectroquant para determinação de cianeto em soluções, nas quantidades adequadas para amostras de $5 \mathrm{~mL}$. A leitura da absorbância a $585 \mathrm{~nm}$ foi feita cinco minutos após a adição dos reagentes, tempo necessário para o desenvolvimento da coloração. O cálculo da quantidade de cianeto liberado, por grama de matéria fresca de casca, foi feito com base em curva padrão de cianeto, nas concentrações de 0,$1 ; 0,2 ; 0,3 ; 0,4$ e $0,5 \mathrm{mg} \mathrm{L}^{-1}$. 


\section{RESULTADOS E DISCUSSÃO}

\section{Resposta à aplicação de $\mathrm{KCN}$ em dose única}

Comparado ao clone IPA 1, o Fx 3864 apresentou tolerância extremamente alta ao $\mathrm{KCN}$, aplicado em diferentes concentrações, em dose única de $2 \mathrm{~mL}$ de solução, tendo o Fx 4098 mostrado comportamento intermediário (TABELA 1). A concentração mais alta de $\mathrm{KCN}$ sem nenhum efeito sobre a exsudação do látex do $\mathrm{Fx} 3864\left(20 \mathrm{mg} \mathrm{L}^{-1}\right)$, com cinco dias após a aplicação, foi 625 vezes maior que a concentração mais baixa $\left(0,032 \mathrm{mg} \mathrm{L}^{-1}\right)$ em que deixou de haver escoamento de látex no IPA 1, em toda a extensão do caule tratado, com dois dias após a aplicação. $O$ volume de solução aplicada $(2 \mathrm{~mL})$ estava totalmente absorvido no dia seguinte ao da aplicação, em todas as concentrações de KCN, exceto no IPA 1, em que houve redução do volume absorvido, progressivamente, a partir da concentração de $4 \mathrm{mg} \mathrm{L}^{-1}$. Mesmo com cinco dias após o início do tratamento, o efeito sobre o escoamento do látex do IPA 1, com a concentração mais alta (100 mg L $\left.{ }^{-1}\right)$, ainda não era completo, o que deve estar relacionado com a menor absorção da solução. Apenas no IPA 1 houve efeito da concentração mais baixa $\left(0,0064 \mathrm{mg} \mathrm{L}^{-1}\right)$, com cinco dias, afetando, porém, pequena extensão do caule. $O$ mesmo grau de resposta foi apresentado pelo Fx 3864, com cinco dias, porém, com a concentração mais alta (100 $\mathrm{mg} \mathrm{L}^{-1}$ ).

Em todas as concentrações de KCN, observouse nos três clones uma faixa, de no máximo $0,5 \mathrm{~cm}$ de largura, de casca necrosada, a partir do topo cortado, próxima do tubo com a solução. Abaixo dessa faixa, mais larga no IPA 1, a casca apresentava aspecto externo sadio. Não houve, portanto reprodução integral dos sintomas verificados com as copas enxertadas incompatíveis com o IPA 1, em que há necrose completa da casca do caule, em certa extensão, abaixo da união do enxerto (Moraes, 1999). A ocorrência de necrose apenas no topo cortado dos ramos laterais sugere que houve bloqueio da translocação de $\mathrm{KCN}$, como relatado por Gur (1957).

$A$ adição de sacarose à solução de $\mathrm{KCN}$ não resultou em aumento da extenção da necrose ao longo do caule, indicando não existir um sistema de cotransporte do cianeto com a sacarose .

\section{Resposta à aplicação contínua de $\mathrm{KCN}$}

Como a aplicação contínua das soluções com diferentes concentrações de $\mathrm{KCN}$, em ramos laterais idênticos aos da aplicação em dose única, a partir do segundo dia a absorção da solução tornou-se muito lenta e foi praticamente nula a partir do terceiro dia.

Em face desse aparente impedimento do transporte do $\mathrm{KCN}$, os resultados da aplicação contínua diferiram muito pouco dos da aplicação em dose única, registrando-se apenas que no Fx 4098 com concentração de $20 \mathrm{mg} \mathrm{L}^{-1}$, a necrose estendeu-se por cerca de $5 \mathrm{~cm}$ abaixo do topo. Tais resultados mostraram a impossibilidade de reprodução da necrose verificada na incompatibilidade de copas enxertadas sobre o IPA 1, devida a pouca mobilidade do KCN na casca do caule dos ramos laterais.

Cerca de 45 dias após os tratamentos houve brotações de gemas ao longo dos caules tratados e recuperação parcial do escoamento do látex, no IPA 1 e no Fx 4098, o que pode ser atribuído à reativação do câmbio.

\section{Resposta à aplicação de linamarina}

O diglicosídio linustatina, sendo a forma natural de transporte (Selmar, 1993) seria o composto ideal para o teste dos efeitos sobre o caule, na tentativa de reprodução dos sintomas de incompatibilidade. Trata-se, entretanto, de composto não disponível comercialmente. Sendo assim, os testes foram feitos com linamarina, que, embora tenha o inconveniente da hidrólise mais rápida que a da linustatina, deveria corresponder a uma forma com maior mobilidade que o KCN.

\section{Marcha de absorção das soluções}

Nos primeiros dois dias, após a aplicação das soluções, houve absorção completa dos $3 \mathrm{~mL}$ aplicados

TABELA 1 - Efeito de doses de KCN, em uma só aplicação, sobre o escoamento do látex no caule.

\begin{tabular}{|c|c|c|c|c|c|c|c|c|c|}
\hline \multirow{3}{*}{$\mathrm{KCN} \mathrm{mg} \mathrm{L}^{-1}$} & \multicolumn{9}{|c|}{ Dias após a aplicação } \\
\hline & \multicolumn{3}{|c|}{1} & \multicolumn{3}{|c|}{2} & \multicolumn{3}{|c|}{5} \\
\hline & IPA 1 & $F \times 4098$ & $F \times 3864$ & IPA 1 & Fx 4098 & $F \times 3864$ & IPA 1 & Fx 4098 & $F \times 3864$ \\
\hline$A-0,0064$ & 0 & 0 & 0 & 1 & 0 & 0 & 1 & 0 & 0 \\
\hline B - 0,032 & 2 & 0 & 0 & 3 & 0 & 0 & 3 & 0 & 0 \\
\hline$C-0,16$ & 2 & 0 & 0 & 3 & 0 & 0 & 3 & 0 & 0 \\
\hline$D-0,8$ & 2 & 0 & 0 & 3 & 0 & 0 & 3 & 0 & 0 \\
\hline$E-4,0$ & 2 & 0 & 0 & 3 & 1 & 0 & 3 & 1 & 0 \\
\hline$F-20,0$ & 2 & 1 & 0 & 2 & 2 & 0 & 3 & 2 & 0 \\
\hline$G-100,0$ & 1 & 1 & 0 & 2 & 2 & 0 & 2 & 3 & 1 \\
\hline
\end{tabular}

0 - Exudação normal a partir das proximidades do topo cortado

1 - Sem exudação até $10 \mathrm{~cm}$ abaixo do topo

2 - Sem exudação até $30 \mathrm{~cm}$ abaixo do topo

3 - Sem exudação até a inserção do ramo (60 a $80 \mathrm{~cm}$ de comprimento). 
diariamente, exceto na dose de $125 \mathrm{mg} \mathrm{L}^{-1}$ no IPA 1. No terceiro dia, houve redução, para cerca da metade do volume aplicado e quase ausência de absorção no IPA 1 , com 25 e $125 \mathrm{mg} \mathrm{L}^{-1}$. No quinto dia, praticamente não houve absorção nos três clones, nas concentrações a partir de $5 \mathrm{mg} \mathrm{L}^{-1}$, enquanto a absorção do controle com água destilada mais DMSO $0,05 \%$, foi, aproximadamente, de $1,2 \mathrm{~mL}$, o que mostra que mesmo sem exposição ao $\mathrm{HCN}$, houve redução, embora mais lenta, da capacidade de transporte do floema, provavelmente apenas mais próximo à superfície cortada.

A marcha da absorção das soluções com linamarina mostra que o sistema de transporte do floema manteve-se funcional por mais tempo que com a exposição direta ao cianeto e destaca, novamente, o IPA 1 como mais sensível, tendo reduzido a absorção em prazo mais curto. Os resultados sugerem, por outro lado, que a hidrólise da linamarina deve ter sido gradual, mesmo no IPA 1, permitindo o transporte, pelo menos de parte, desse glicosídio, em maior extensão que a do $\mathrm{KCN}$, ao longo do caule, a julgar pelos efeitos sobre o escoamento e indução de necrose, relatados a seguir.

\section{Efeito sobre o escoamento do látex e indução de necrose}

Os resultados mostram claramente que a coagulação do látex nos laticíferos precede o aparecimento da necrose da casca visível externamente (TABELA 2), tal como acontece quando copas incompatíveis são enxertadas sobre o IPA 1, porém em intervalos mais curtos.

Com dois dias após o início do tratamento, deixou de haver escoamento do látex no IPA 1, em todo o comprimento do ramo lateral tratado, exceto na concentração mais baixa e na mais alta. Certamente, o menor efeito da concentração mais alta, com dois dias no IPA 1, foi devido ao bloqueio da translocação já mencionado, o que implica em maior concentração de $\mathrm{HCN}$ livre nesse clone, com a aplicação de linamarina. Com quatro dias verificou-se maior extensão do caule sem escoamento de látex, no IPA 1 , com a dose mais baixa, ocorrendo o mesmo com a dose mais alta, no sexto dia (TABELA 2).

Como na aplicação de KCN, a ausência de escoamento de látex com aplicação de linamarina manifestou-se mais cedo e de modo mais severo no IPA 1 que no $F x$ 4098, enquanto o Fx 3864 não foi afetado. No Fx 4098 as duas doses mais baixas também não causaram coagulação do látex. Esse efeito foi apenas parcial, com a concentração de $1 \mathrm{mg} \mathrm{L}^{-1}$, ainda não atingindo todo o comprimento do ramo lateral tratado com $5 \mathrm{mg} \mathrm{L}^{-1}$. Nas duas concentrações mais altas observou-se ausência de escoamento em todo o comprimento do ramo lateral.

Entre a coagulação do látex e os sintomas externamente visíveis de necrose da casca, houve um intervalo de quatro dias no IPA 1 e de seis dias no Fx 4098, o que contrasta com o período de até dois meses, entre o início da redução do escoamento do látex e os

TABELA 2 - Efeito de doses crescentes de linamarina sobre o escoamento do látex e indução de necrose em toda a espessura da casca, aplicadas durante cinco dias consecutivos nos clones IPA 1, Fx 4098 e Fx 3864.

\begin{tabular}{|c|c|c|c|c|c|c|c|c|c|c|c|c|}
\hline \multirow{3}{*}{$\begin{array}{l}\text { Linamarina } \\
\mathrm{mg} \mathrm{L}^{-1}\end{array}$} & & & & \multicolumn{6}{|c|}{ Dias após a aplicação } & & & \\
\hline & \multicolumn{3}{|c|}{2} & \multicolumn{3}{|c|}{4} & \multicolumn{3}{|c|}{6} & \multicolumn{3}{|c|}{10} \\
\hline & $\begin{array}{c}\text { IPA } \\
1\end{array}$ & $\begin{array}{c}F x \\
4098\end{array}$ & $\begin{array}{c}F x \\
3864\end{array}$ & $\begin{array}{c}\text { IPA } \\
1\end{array}$ & $\begin{array}{c}\text { Fx } \\
4098\end{array}$ & $\begin{array}{c}F x \\
3864\end{array}$ & $\begin{array}{c}\text { IPA } \\
1\end{array}$ & $\begin{array}{c}F x \\
4098\end{array}$ & $\begin{array}{c}F x \\
3864\end{array}$ & $\begin{array}{c}\text { IPA } \\
1\end{array}$ & $\begin{array}{c}F x \\
4098\end{array}$ & $\begin{array}{c}F x \\
3864\end{array}$ \\
\hline \multirow{2}{*}{ A- 0,04} & - & - & - & - & - & - & $\mathrm{N}$ & - & - & NNN & - & - \\
\hline & 1 & 0 & 0 & 3 & 0 & 0 & 3 & 0 & 0 & 3 & 0 & 0 \\
\hline \multirow{2}{*}{ B $-0,2$} & - & - & - & - & - & - & NN & - & - & NNN & - & - \\
\hline & 3 & 0 & 0 & 3 & 0 & 0 & 3 & 0 & 0 & 3 & 0 & 0 \\
\hline \multirow{2}{*}{$C-1,0$} & - & - & - & - & - & - & NN & - & - & NNN & - & - \\
\hline & 3 & 0 & 0 & 3 & 0 & 0 & 3 & 1 & 0 & 3 & 1 & 0 \\
\hline \multirow{2}{*}{$D-5,0$} & - & - & - & - & - & - & NN & - & - & NNN & - & - \\
\hline & 3 & 0 & 0 & 3 & 2 & 0 & 3 & 2 & 0 & 3 & 2 & 0 \\
\hline \multirow{2}{*}{$E-25,0$} & - & - & - & - & - & - & $N$ & - & - & $\mathrm{NN}$ & - & - \\
\hline & 2 & 0 & 0 & 3 & 3 & 0 & 3 & 3 & 0 & 3 & 3 & 0 \\
\hline \multirow{2}{*}{$F-125,0$} & - & - & - & - & - & - & $\mathrm{N}$ & - & - & $\mathrm{NN}$ & - & - \\
\hline & 1 & 1 & 0 & 2 & 2 & 0 & 3 & 3 & 0 & 3 & 3 & 0 \\
\hline \multirow{2}{*}{$\begin{array}{l}\text { DMSO } \\
(0,05 \%)\end{array}$} & - & - & - & - & - & - & - & - & - & - & - & - \\
\hline & 0 & 0 & 0 & 0 & 0 & 0 & 0 & 0 & 0 & 0 & 0 & 0 \\
\hline $\begin{array}{l}\text { : : Ausência } \\
\text { N: Necrose até } \\
\text { NN: Necrose at } \\
\text { NNN: Necrose }\end{array}$ & $\begin{array}{l}\text { crose } \\
n \text { abai } \\
\mathrm{cm} \text { abs } \\
\text { do cor }\end{array}$ & $\begin{array}{l}\text { xo do to } \\
\text { orimente }\end{array}$ & do ramo & $80 \mathrm{cr}$ & & $\begin{array}{l}0: \text { Es } \\
1: \text { Se } \\
2: \text { Ser } \\
3: \text { Ser }\end{array}$ & $\begin{array}{l}\text { ento } \\
\text { coame } \\
\text { oame } \\
\text { oame }\end{array}$ & $\begin{array}{l}\text { mal, a } \\
\text { o a até } 10 \\
\text { o até } 30 \\
\mathrm{~b} \text { em toc }\end{array}$ & $\begin{array}{l}\text { rtir da } p \\
\text { cm abai } \\
\text { m abaix } \\
\text { o comp }\end{array}$ & $\begin{array}{l}\text { nidade } \\
\text { o topo } \\
\text { topo } \\
\text { into do r }\end{array}$ & amo $(6$ & $80 \mathrm{~cm})$ \\
\hline
\end{tabular}

Scientia Agricola, v.58, n.4, p.717-723, out./dez. 2001 
primeiros sinais externos de necrose da casca do IPA 1 quando enxertado com copas incompatíveis. Tal fato, em princípio, deve ser atribuído à rapidez da liberação de HCN pela linamarina, sob ação da $\beta$-glicosidase situada nas paredes das células.

A necrose da casca do caule, verificada aos dez dias, no Fx 4098, foi provocada apenas pelas duas concentrações mais altas de linamarina, com a necrose estendendo-se entre 10 e $30 \mathrm{~cm}$ abaixo do topo cortado. No Fx 3864 nenhuma das concentrações de linamarina testadas causou necrose da casca, o que é coerente com a ausência de efeito sobre o escoamento do látex.

Verifica-se assim que o Fx 3864 mostrou-se insensível às doses de $\mathrm{KCN}$ e de linamarina, o que poderia ser atribuído a maior imobilidade do $\mathrm{KCN}$ e da linamarina nesse clone, mas, nesse caso, teria ocorrido necrose nítida próximo à superfície do topo cortado, em contato imediato com as soluções. Como hipótese alternativa, pode-se conjecturar uma maior capacidade de fixação do HCN no Fx 3864 e, um pouco menor, no Fx 4098, comparada a capacidade muito baixa de desintoxicação do IPA 1 o que poderia ser comprovado pela determinação dos níveis de atividade da $\beta$ cianoalaninasintase nesses três clones (Lieberei, 1988).

\section{Ação do cianeto na coagulação do látex "in vitro"}

A coagulação do látex nos laticíferos do IPA 1, em prazo mais curto de tratamento com as soluções de $\mathrm{KCN}$ ou linamarina aplicadas no caule, que dos outros dois clones, não é devida a menor estabilidade do látex do IPA 1 ao cianeto (TABELA 3). Pelo contrário, verificouse que foi necessário um volume maior de solução de $\mathrm{KCN}, 300 \mathrm{mmol} \mathrm{L}^{-1}$, para a coagulação do látex do IPA 1.

\section{Concentração de HCN livre nos caules submetidos a diferentes tempos de exposição à solução de linamarina}

Os efeitos da aplicação de KCN e de linamarina, sobre o escoamento do látex e indução de necrose, indicam maior concentração de $\mathrm{HCN}$ livre na casca do caule do IPA 1, enquanto o Fx 3864 praticamente não foi afetado pelos tratamentos com $\mathrm{KCN}$, ou linamarina (Figura 1). Esses resultados mostraram-se plenamente coerentes com a previsão. Observa-se que houve acúmulo de HCN no IPA 1 até 12 horas após o início do tratamento. A redução gradativa para teores abaixo dos encontrados no Fx 3864, com 24 horas após o início do tratamento, deve ser atribuída à degeneração dos tecidos da casca pela ação tóxica do cianeto, que inibe a cadeia de transporte eletrônico da respiração normal, sendo a energia metabolicamente disponível, gerada pelo caminho alternativo da respiração resistente ao cianeto, caracterizada na casca da seringueira por Siew \& Shaoqiong (1984), insuficiente para a síntese de compostos complexos ou para a manutenção da integridade das membranas.
TABELA 3 - Volume da solução de $\mathrm{KCN}, 300 \mathrm{mmol} \mathrm{L}^{-1}$, consumido na coagulação de $20 \mathrm{~mL}$ de látex.

\begin{tabular}{lc}
\hline Clone & $\mathrm{KCN} 300 \mathrm{mmol} \mathrm{L}^{-1}(\mathrm{~mL})$ \\
\hline Fx 4098 & 1,2 \\
Fx 3864 & 1,7 \\
IPA 1 & 2,1 \\
\hline
\end{tabular}

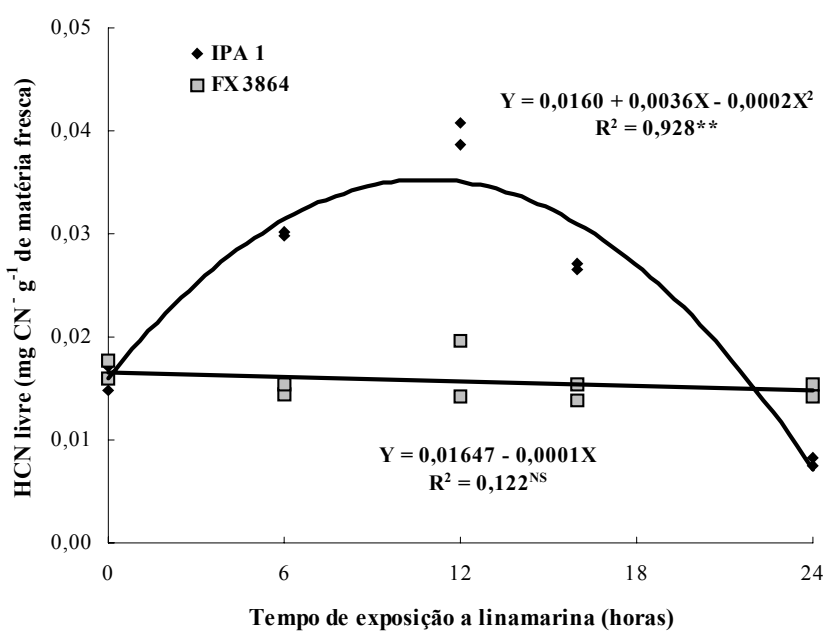

Figura 1 - HCN livre na casca do caule dos clones IPA 1 e Fx 3864 em $\mathrm{mg} \mathrm{g}^{-1}$ de matéria fresca, em função do tempo de exposição a dose única de solução de linamarina $(6 \mathrm{mg}$ $\left.\mathrm{L}^{-1}\right)$. ${ }^{\star}$ Significativo a $1 \%$ de probabilidade pelo teste $\mathrm{F}$ e ${ }^{N S}$ não significativo.

No Fx 3864 o teor de HCN livre permaneceu constante durante o período das observações, devendose, entretanto, considerar a possibilidade de que parte do HCN liberado seja produto de ação residual da $\beta$ glicosidase, no $\mathrm{pH} 2,81$, observado nas amostras extraídas com o ácido ortofosfórico, conforme a curva de atividade em função do $\mathrm{pH}$, apresentada por Selmar (1986), o mesmo sendo válido para os extratos das amostras de casca do IPA 1. De qualquer modo, o maior teor de HCN livre no IPA 1 está de acordo com as respostas ao $\mathrm{KCN}$ e linamarina, considerando-se, por outro lado, que uma provável alta atividade de $\beta$ cianoalaninasintase nesse clone deveria atuar no sentido de manutenção de baixos teores de HCN livre. Os resultados obtidos com as aplicações de $\mathrm{KCN}$ e linamarina indicam a necessidade de estudo das enzimas envolvidas na cianogênese e na refixação do $\mathrm{HCN}$.

\section{CONCLUSÕES}

A extrema sensibilidade do clone de painel IPA 1 às substâncias cianogênicas aplicadas reforça a hipótese de a incompatibilidade apresentada por esse clone enxertado com clones de copa de outras espécies de Hevea é devida à translocação de glicosídios cianogênicos da copa para o caule do IPA 1, com liberação de $\mathrm{HCN}$, atingindo níveis tóxicos. 


\section{REFERÊNCIAS BIBLIOGRÁFICAS}

BLUMENTHAL, S.G.; HENDRICKSON, H.R.; ABROL, Y.P.; CONN, E.E. Cyanide metabolism in higher plants: III. The biosynthesis of $\beta$-cyanoalanine. Journal of Biological Chemistry, v.243, p.5302-5307, 1968.

CASTRIC, P.A.; FARNDEN, K.J.F.; CONN, E.E. Cyanide metabolism in higher plants. $V$. The formation of asparagine from $\beta$-cyanoalanine. Archives of Biochemistry and Biophysics, v.152, p.62-69, 1972.

CONN, E.E. Cyanogenic compounds. Annual Review of Plant Physiology, v.31, p.433-451, 1980.

COOKE, R.D. An enzymatic assay for the total cyanide content of cassava (Manihot esculenta Crantz). Journal of the Science of Food and Agriculture, v.29, p.345-352, 1978.

FLOSS, H.G.; HADWIGER, L.; CONN, E.E. Enzymatic formation of $\beta$-cyanoalanine from cyanide. Nature, v.208, p.1207-1208, 1965.

GUR, A. The incompatibility of the pear with quince rootstock. Israel Ministry of Agriculture Research Station, 1957. 36p. (Special Bulletin, 10)

HARTMANN, T.H.; KESTER, D.E.; DAVIES JR., F.T. Plant propagation: principles and practices. 5.ed. New Jersey: Englewood Cliff, 1990. 647p.

KAKES, P. Properties and functions of the cyanogenic system in higher plants. Euphytica, v.48, p.25-43, 1990.

LIEBEREI, R.; NAHRSTED, A.; SELMAR, D.; GASPAROTTO, L. Occurrence of lotaustralin in the genus Hevea and of HCNpotential in developing organs of Hevea brasiliensis Phytochemistry, v.25, p.1573-1578, 1986.

LIEBEREI, R. Cyanogenesis of Hevea brasiliensis during infection with Mycrocyclus ulei. Journal of Phytopathology, v.115, p.134-146, 1986.
LIEBEREI, R. Relationship of cyanogenic capacity (HCN-c) of the rubber tree Hevea brasiliensis to susceptibility to Microcyclus ulei, the agent causing South American leaf blight. Journal of Phytopathology, v.122, p.54-67, 1988.

MILLER, J.M.; CONN, E.E. Metabolism of hydrogen cyanide by higher plants. Plant Physiology, v.65, p.1199-1202, 1980.

MORAES, V.H.F. Enxertia de copa para remoção dos impedimentos naturais a heveicultura na Amazônia sempre úmida: Subprojeto (070.94.018.01) ; Relatório final. Manaus: Embrapa Amazônia Ocidental, 1999. 12p. (Programa 07 - Sistema de Produção de Matérias Primas).

MORAES, V.H.F. Sumário das pesquisas sobre enxertia de copa da seringueira no CPAA. Manaus: Embrapa, CPAA, 1997. 25p. (Documentos, 8).

MOSSE, B. Graft-incompatibility in fruit trees. East Mailing Bureau of Horticulture, 1962. 36p. (Technical Communication, 28)

SELMAR, D. Transport of cyanogenic glucosides linustatin uptake by Hevea cotyledons. Planta, v.191, p.191-198, 1993.

SELMAR, D. Cyanogenese in Hevea. Zwei wege zur metabolisierung cyanogener glucoside. Braunschweig, 1986. 171p. Doktorarbeit (Doktorarbeit) - Technischey Universität Braunschweig.

SELMAR, D.; GROCHOLEWSKI, S.; SEIGLER, D.S. Cyanogenic lipids: Utilization during seedling development of Ungnadia speciosa. Plant Physiology, v.93, p.631-636, 1990.

SIEW, F.; SHAOQIONG, Y. Preliminary study of cyanide resistant respiration in Hevea brasiliensis. In: COLLOQUE EXPLOTATION PHYISIOLOGIE ET AMÉLIORATION DE L'HEVEA, Montpellier, 1984. Compte-Rendu. Montpellier: IRCA/GERDAT, 1984. p.185-203.

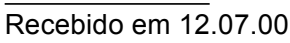

